\title{
Well-Deserved Credit for Bipolar Disorder
}

\author{
By Jack M. Gorman, MD
}

At one point less than a decade ago, I was surprised to learn from the then director of the National Institute of Mental Health (NIMH), Steven Hyman, MD, that his agency was funding no clinical trials involving patients with bipolar disorder. Dr. Hyman quickly corrected that situation (more on that in a moment), but it is worthwhile to pause for a moment and consider why one of psychiatry's most devastating illnesses was being left untouched by the researcher's hand.

At first glance, bipolar disorder would seem to be an ideal illness to study. In its classical form, it is not difficult to diagnose, the patient has periods of normal mood and behavior, it is highly familial, and a specific drug-lithium - treats it. These clues ought to be firm guideposts for pinpointing the pathophysiology of the illness. Yet, for a number of reasons, bipolar disorder has not been considered attractive enough to warrant study.

First, the diagnosis is, in fact, often quite difficult. Patients with bipolar illness often had several depressive episodes before the first manic one and, therefore, for long periods of time they are given a diagnosis of recurrent depression rather than bipolar disorder.

Then, there are the many forms of bipolar disorder-bipolar I, bipolar II, cyclothymia, rapid cycling, etc. These variations make diagnosis difficult and complicate family studies. Also, bipolar patients are often difficult to manage in a research setting. When depressed, they often become suicidal and cannot be kept in a clinical trial. When manic, they refuse to take medication and can quickly exhaust the time and patience of clinical staff. And lithium - one of the greatest medical advances of the 20th century-has a mechanism of action that has remained almost entirely obscure.
Therefore, researchers have tended to direct their attention to schizophrenia, depression, anxiety disorders, and eating disorders-almost everything except bipolar disorder. That situation is a great disservice to the $\sim 1 \%$ of the population who suffer with bipolar disorder and their families.

Recently, data has been reported from the huge NIMH-funded study of bipolar patients known as Systematic Treatment Enhancement Program for Bipolar Disorder (STEP-BD). In this study, an attempt has been made to enter thousands of bipolar patients into an effectiveness study examining the relative methods of several different treatments of the disorder. Perhaps equally important is that these well-characterized patients were asked to consent to being genotyped so that genetic information will be available to researchers on the largest cohort of bipolar patients yet assembled. The field is eagerly awaiting the main articles from STEP-BD, but already say it can be stated with confidence that the fact that the study was funded indicates a sea change in our attitude toward bipolar disorder research.

The same can be said of the researchers represented in this issue of CNS Spectrums. Assembled by distinguished investigator Stephen M. Strakowski, MD of the University of Cincinnati, in Ohio, these articles represent the very best in neuroimaging of bipolar disorder. To be sure, the work does not easily lend itself to a unified view of exactly what is wrong in the brains of patients with bipolar disorder. Findings differ among studies, probably in large part because of the great phenotypic heterogeneity among patients with bipolar disorder. Nevertheless, some clear patterns begin to unfold. Coupled with what is coming in the genetic area, we can finally be confident that bipolar disorder is getting its fair share of attention. CNS

Dr. Gorman is the editor of this journal, president and psychiatrist-in-chief at Mclean Hospital, chair of Partners Psychiatry and Mental Health, both in Belmont, Massachusetts, and professor of psychiatry at Harvard Medical School in Boston. 